\title{
ON DEFORMATIONS OF HOMOMORPHISMS OF LOCALLY COMPACT GROUPS
}

\author{
BY
}

\section{DONG HOON LEE}

ABSTRACT. The rigidity of homomorphisms of compactly generated locally compact groups into Lie groups is investigated.

For locally compact groups $G$ and $H$, let $\operatorname{Hom}(G, H)$ denote the space of all continuous homomorphisms of $G$ into $H$ under the compact-open topology. Then $H$ acts continuously on the space $\operatorname{Hom}(G, H)$ under conjugation. We say that $f \in \operatorname{Hom}(G, H)$ is rigid if the orbit of $f$ in $\operatorname{Hom}(G, H)$ under the action of $H$ is an open neighborhood of $f$. In [6], Nijenhuis and Richardson have obtained the rigidity of homomorphisms of real analytic groups (or more generally, compactly generated Lie groups) into any Lie groups under certain cohomological conditions, thereby generalizing the well-known theorem of Weil [7], and conjectured that their result can be generalized to compactly generated, locally compact groups.

The main purpose of this paper is to prove this conjecture affirmatively. Our proof consists mainly of the reduction to the case of Lie groups. $\$ 1$ carries basic definitions and notation which are standard throughout. In $\$ 2$, we embed the space Hom $(G, H)$ as a closed analytic subset of a manifold. In $\$ 3$ we prove the result of Nijenhuis and Richardson, the proof of which does not seem to have appeared at the time of preparation of this paper. Finally $\$ 4$ carries the proof of our main result together with some of its applications and an example.

We wish to thank T. S. Wu for helpful discussions during the preparation of this paper.

1. Basic definition and notation.

(1.1) Let $G$ be a locally compact group and let $\rho$ be a continuous representation of $G$ in a finite-dimensional real vector space $V$. A continuous map $\phi: G \rightarrow V$ is called a 1-cocycle of $G$ with values in $V$ (relative to $\rho$ ) if, for $x, y \in G, \phi(x y)=\phi(x)+\rho(x)(\phi(y))$. The set of all l-cocycles with values in $V$ forms a vector space, which we denote by $Z^{1}(G, V, \rho)$. A cocycle $\phi \in Z^{1}(G, V, \rho)$

Received by the editors June 27, 1973.

AMS (MOS) subject classifications (1970). Primary 22D05, 22E15, 22E40.

Key words and phrases. Rigidity, compactly generated, cohomology space, exponential maps, p-adic integers.

Copyright $\odot$ 1974, American Mathematical Society 
is called a 1-coboundary, if there exists $v \in V$ so that $\phi(x)=\rho(x)(v)-v$ for all $x \in G$. The set of all 1-coboundaries forms a subspace $B^{1}(G, V, \rho)$. Let $H^{1}(G, V, \rho)=Z^{1}(G, V, \rho) / B^{1}(G, V, \rho)$ and call it the 1-cobomology space of $G$ with coefficients in $V$. For a detailed discussion, see [5].

(1.2) Let $G$ and $H$ be locally compact groups. Then the set $\operatorname{Hom}(G, H)$ becomes a topological space under the compact-open topology. To describe neighborhoods of $f_{0} \in \operatorname{Hom}(G, H)$, let $C$ be a compact subset of $G$ and let $U$ be a 1 -neighborhood in $H$. Then define

$$
W\left(C, U ; f_{0}\right)=\left\{f \in \operatorname{Hom}(G, H): f(x) f_{0}(x)^{-1} \in U \text { for all } x \in C\right\} .
$$

When $C$ and $U$ run over all compact subsets of $G$ and all 1-neighborhoods in $H$, respectively, the sets $W\left(C, U, f_{0}\right)$ form a neighborhood basis of $f_{0}$ in $\operatorname{Hom}(G, H)$. If $G$ and $H$ are both connected Lie groups with their Lie algebras $\biguplus$ and $\mathcal{H}$, respectively, then we have a natural embedding of $\operatorname{Hom}(G, H)$ into $\operatorname{Hom}\left(\mathcal{H}_{,} \mathcal{H}\right)$ via $f \rightarrow d f$, where $d f$ is the differential of $f$ at 1 , being identified with the Lie algebra homomorphism induced from $f$.

(1.3) The following notation is standard throughout. For any topological group $H$, let $H_{0}$, Aut $(H)$ denote the 1-component, the automorphism group of $H$, respectively. Also for each $x \in H, I_{x}$ denotes the inner automorphism of $H$ induced by $x$, and, for $X \subset H$, Int ${ }_{H}(X)=\left\{I_{x}: x \in X\right\}$. If $X=H$, then we write Int $(H)$ for $\operatorname{Int}_{H}(H)$. Then $H$ acts on $\operatorname{Hom}(G, H)$ via $(t, f) \rightarrow I_{x} \circ f$, and $f \epsilon$ Hom $(G, H)$ is rigid if Int $(H), f$ is an open neighborhood of $f$ in $\operatorname{Hom}(G, H)$. If $H$ is a $\mathrm{Lie}$ group, $\mathrm{Ad}_{H}$ denotes the adjoint representation of $H$ in the Lie algebra of $H$.

Finally, a Lie group and its Lie algebra are denoted by the same capital italic and capital English script letter, respectively. Thus, for example, if $G$ is a Lie group, then $\subseteq$ denotes the Lie algebra of $G$.

2. The embedding of Hom $(G, H)$ into a manifold.

(2.1) Let $G$ be a compactly generated Lie group. We choose $\gamma_{1}, \ldots, \gamma_{m} \in G$ once and for all so that the $\gamma_{j}$ generate $G$ modulo $G_{0}$. Let $F$ be the free group on symbols $\tilde{y}_{1}, \ldots, \tilde{\gamma}_{m}$ and let $\sigma: F \rightarrow G$ be the homomorphism determined by $\sigma\left(\tilde{\gamma}_{j}\right)=\gamma_{j}$.

For any Lie group $H$, let $i^{*}=\operatorname{Hom}(i, 1): \operatorname{Hom}(G, H) \rightarrow \operatorname{Hom}\left(G_{0}, H\right)$ and $\sigma^{*}=\operatorname{Hom}(\sigma, 1): \operatorname{Hom}(G, H) \rightarrow \operatorname{Hom}(F, H)$ be the dual maps induced by the inclusion $i: G_{0} \rightarrow G$ and $\sigma$, respectively.

Then clearly $i^{*} \times \sigma^{*}: \operatorname{Hom}(G, H) \rightarrow \operatorname{Hom}\left(G_{0}, H\right) \times \operatorname{Hom}(F, H)$ is a continuous injection. To determine $\operatorname{Im}\left(i^{*} \times \sigma^{*}\right)$, let $\left(f_{0}, g\right) \in \operatorname{Hom}\left(G_{0}, H\right) \times \operatorname{Hom}(F, H)$ and define $f(x \sigma(\gamma))=f_{0}(x) g(y)$ for $(x, \gamma) \in G_{0} \times F$. Noting that $G=G_{0} \cdot \operatorname{Im} \sigma$, we have 
(1)

$f$ defines a map $f: G \rightarrow H$ if and only if

$$
f_{0} \sigma(\gamma)=g(\gamma) \text { whenever } \sigma(\gamma) \in G_{0} \text {. }
$$

Since the $\gamma_{j}$ generate $F, f$ is a homomorphism if and only if

$$
f_{0} \cdot I_{\gamma_{j}}=I_{\boldsymbol{g}\left(\gamma_{j}\right)} \cdot f_{0} \quad(1 \leq j \leq m)
$$

Let $\left(\omega_{\lambda}\right)_{\lambda \in \Lambda}$ be an indexed set of generators of the subgroup $\sigma^{-1}\left(G_{0} \cap \operatorname{Im} \sigma\right)$ of $F$ and put $a_{\lambda}=\sigma\left(\omega_{\lambda}\right), \lambda \in \Lambda$.

Then the condition in (1) can be replaced by

$$
f_{0}\left(a_{\lambda}\right)=g\left(\omega_{\lambda}\right) \text { for } \lambda \in \Lambda \text {. }
$$

Hence $\left(f_{0}, g\right) \in \operatorname{Im}\left(i^{*} \times \sigma^{*}\right)$ if and only if it satisfies conditions (3) and (2).

(2.2) Now we choose a basis $\left(X_{i \leq i \leq n}\right.$ of $\mathcal{Y}$ once and for all and let $x_{1}, \ldots, x_{n}$ be a canonical system of coordinates for a fixed exponential map $\exp _{G}: \mathcal{G} \rightarrow G_{0}$ defined on a 1 -neighborhood $V$ of $G$ as in $[1, \mathrm{p} .118]$. Thus if $a \in V$, then $a=\exp \left(\sum_{k=1}^{n} x_{k}(a) X_{k}\right)$. Since $V$ generates $G_{0}$, the elements $a_{\lambda}=$ $\sigma\left(\omega_{\lambda}\right)$ may be written in the form

$$
a_{\lambda}=a_{\lambda, 1}, \cdots, a_{\lambda, l(\lambda)} \text { with } a_{\lambda, i} \in V(1 \leq i \leq l(\lambda)) .
$$

Hence

$$
a_{\lambda}=\prod_{i=1}^{l(\lambda)} \exp \left(\sum_{k=1}^{n} x_{k}\left(a_{\lambda, i}\right) X_{k}\right)
$$

On the other hand, every element $\omega_{\lambda}$ can be written in the form

$$
\omega_{\lambda}=\gamma_{i_{1}}^{\epsilon_{1}} \cdots \gamma_{i_{k(\lambda)}}^{\epsilon_{k(\lambda)}}, \quad \epsilon_{i}= \pm 1,
$$

where $\left(i_{1}, \ldots, i_{k(\lambda)}\right)$ is a subset of $(1,2, \ldots, m)$.

We fix one such expression and define $W_{\lambda}: H^{m} \rightarrow H$ by $W_{\lambda}\left(b_{1}, \ldots, b_{m}\right)=$ $b_{i_{1}}^{\epsilon_{1}} \ldots b_{i_{k(\lambda)}}^{\epsilon_{k(\lambda)}}$. Thus, using $\exp _{H} \cdot d f=f \cdot \exp _{G}$, we may replace (3) by

$$
\prod_{i=1}^{l(\lambda)} \exp _{H}\left(\sum x_{k}\left(a_{\lambda, i}\right) d f\left(x_{k}\right)\right)=W_{\lambda}\left(g\left(\gamma_{1}\right), \ldots, g\left(\gamma_{m}\right)\right) \text {. }
$$

(2.3) Let $r: \tilde{G}_{0} \rightarrow G_{0}$ be the universal covering of $G_{0}$ and let $\tilde{x}_{1}, \ldots, \tilde{x}_{n}$ be a canonical system of coordinates defined on a 1 -neighborhood $\tilde{V}$ of $\tilde{G}_{0}$ so" that every $\tilde{a} \in \tilde{V}$ can be written in the form

$$
\tilde{a}=\exp \left(\sum_{k=1}^{n} \tilde{x}_{k}(\tilde{a}) X_{k}\right) \text {. }
$$

It is well known that ker $r$ is finitely generated. Let $\left(\tilde{b}_{1}, \ldots, \tilde{b}_{l}\right)$ be a set of generators of $\operatorname{ker} \tau$. Since $\tilde{V}$ generates $\tilde{G}_{0}$, there exist $\tilde{b}_{j, 1}, \ldots, \tilde{b}_{j, m(j)} \in V$ so that 


$$
\tilde{b}_{j}=\prod_{p=1}^{m(j)} \tilde{b}_{j, p}=\prod_{p=1}^{m(j)} \exp \left(\sum_{k=1}^{n} x_{k}\left(b_{j, p}\right) X_{k}\right) .
$$

Now consider the dual map $r^{*}=\operatorname{Hom}(r, 1): \operatorname{Hom}\left(G_{0}, H\right) \rightarrow \operatorname{Hom}\left(\tilde{G}_{0}, H\right)$. Clearly $f_{0} \in \operatorname{Hom}\left(G_{0}, H\right)$ belongs to $\operatorname{Im} r^{*}$ if and only if $f_{0}(\operatorname{Ker} r)=1$.

Hence we have $f_{0} \in \operatorname{Im} r^{*}$ if and only if it satisfies

$$
\prod_{p=1}^{m(j)} \exp _{H}\left(\sum_{k=1}^{n} \tilde{x}_{k}\left(\tilde{b}_{j, p}\right) d f_{0}\left(x_{k}\right)\right)=1 \quad(1 \leq j \leq l) .
$$

(2.4) We now define $\epsilon: \operatorname{Hom}(G, H) \rightarrow \mathcal{H}^{n} \times H^{m}$ by

$$
\epsilon(f)=\left(d f\left(X_{1}\right), \ldots, d f\left(X_{m}\right), f\left(\gamma_{1}\right), \ldots, f\left(\gamma_{m}\right)\right)
$$

Note that $\epsilon$ is merely the composition of the following sequence of maps:

$\operatorname{Hom}(G, H) \rightarrow \operatorname{Hom}\left(G_{0}, H\right) \times \operatorname{Hom}(F, H) \rightarrow \operatorname{Hom}\left(\tilde{G}_{0}, H\right) \times H^{m} \rightarrow \simeq \operatorname{Hom}\left(\mathcal{G}_{0} \mathcal{H}\right) \times$ $H^{m} \rightarrow \mathcal{H}^{n} \times \mathcal{H}^{m}$, where $\operatorname{Hom}\left(G_{0}, H\right) \cong \operatorname{Hom}(\mathcal{G}, \mathcal{H})$ is given by $f \rightarrow d f$.

From this, it is easy to see that $\epsilon: \operatorname{Hom}(G, H) \rightarrow \operatorname{Im} \epsilon$ is a homeomorphism.

Using the formulas (3), (4) and (5), we have

Lemma. $\left(Y_{1}, \cdots, Y_{n} ; b_{1}, \ldots, b_{m}\right) \in \mathcal{G}^{n} \times H^{m}$ is in $\operatorname{Im} \epsilon$ if and only if it satisfies the following conditions:

(i) $\Pi_{p=1}^{m(j)} \exp _{H}\left(\Sigma \tilde{x}_{k}\left(\tilde{b}_{j, p}\right) Y_{k}\right)=1 \quad(1 \leq j \leq l)$.

(ii) $\sum_{k=1}^{n} C_{i j k} Y_{k}-\left[Y_{i}, Y_{j}\right]=0(1 \leq i, j \leq n)$, where the $C_{i j k}$ are the struc. tural constants of $\mathrm{G}$.

(iii) Ad $\left(b_{j}\right)\left(Y_{i}\right)-\sum_{k=1}^{n} r_{i k}^{j} Y_{k}=0(1 \leq i \leq n, 1 \leq j \leq m)$, where $\left(r_{i k}^{j}\right)_{i, k}$ is the matrix of $\operatorname{Ad}\left(\gamma_{j}\right): @ \rightarrow \varrho$ relative to the basis $X_{i}$.

(iv) $\Pi_{i=1}^{l a} \exp \left(\Sigma_{k} x_{k}\left(a_{\lambda, i}\right) Y_{k}\right) W\left(b_{1}, \cdots, b_{m}\right)^{-1}=1$ for $\lambda \in \Lambda$.

Define $\Phi_{j}\left(Y_{1}, \cdots, Y_{n} ; b_{1}, \ldots, b_{m}\right), \psi_{i j}\left(Y_{1}, \ldots, Y_{n} ; b_{1}, \cdots, b_{m}\right)$, $\Omega_{i j}\left(Y_{1}, \ldots, Y_{n} ; b_{1}, \ldots, b_{m}\right)$ and $\chi_{\lambda}\left(Y_{1}, \ldots, Y_{n} ; b_{1}, \ldots, b_{m}\right)$ to be the expressions on the left-hand sides of the equations (i), (i), (ii), (iii) and (iv), respectively.

Then $\Phi_{j}, \chi_{\lambda}$ and $\psi_{i j}, \Omega_{i j}$ are all $C^{\infty}$-maps of $\mathcal{H}^{n} \times H^{m}$ into $H$ and $\mathcal{H}$, respectively.

3. Deformations of Lie groups.

(3.1) Let $\rho: G \rightarrow$ Aut $(V)$ be a continuous representation in a finite-dimensional real vector space $V$. We define an embedding $\epsilon^{\prime}: Z^{1}(G, V, \rho) \rightarrow V^{n} \times V^{m}$ by $\epsilon^{\prime}(f)=\left(d f\left(X_{1}\right), \cdots, d f\left(X_{m}\right) ; f\left(\gamma_{1}\right), \cdots, f\left(\gamma_{m}\right)\right)$. Here we identified $V$ with the tangent linear space of $V$ at 0 .

We give a convenient description of $\operatorname{Im}\left(\epsilon^{\prime}\right)$ below. For this purpose, we take the semidirect product $V \times{ }_{\rho} G$ of $V$ by $G$ relative to $\rho$.

For any map $f: G \rightarrow V$, define $f^{\prime}: G \rightarrow V \times{ }_{\rho} G$ by $f^{\prime}(x)=(f(x), x)$. Then 
$f \in Z^{1}(G, V, \rho)$ if and only if $f^{\prime} \in \operatorname{Hom}\left(G, V \times_{\rho} G\right)$. Applying the lemma in $\$ 2$ to $f^{\prime}$ with $H=V \times{ }_{\rho} G$, it is not difficult to obtain

Lemma. $\left(v_{1}, \ldots, v_{n} ; u_{1}, \ldots, u_{m}\right) \in V^{n} \times V^{m}$ is in $\operatorname{Im}\left(\epsilon^{\prime}\right)$ if and only if it satisfies the following:

$$
\sum_{p=1}^{m(j)} \sum_{k=1}^{n} \beta_{j, p, k}=0 \quad(1 \leq j \leq l),
$$

where $\beta_{j, p, k}=\tilde{x}_{k}\left(\tilde{b}_{j, p}\right) \rho\left(\tilde{b}_{j, 1} \ldots \tilde{b}_{j, p-1}\right) v_{k}$ for $1 \leq k \leq n, 1 \leq p \leq m(j)$.

$$
\sum_{k=1}^{n} c_{i j k} X_{k}-d \rho\left(X_{j}\right)\left(v_{i}\right)+d \rho\left(X_{i}\right)\left(v_{j}\right)=0 \quad(1 \leq i, j \leq n) .
$$

(iii) $\rho\left(\gamma_{j}\right) v_{i}-\rho\left(\gamma_{j}\right) \cdot d \rho\left(X_{i}\right) \cdot \rho\left(\gamma_{j}\right)^{-1}\left(u_{j}\right)-\sum_{k=1}^{n} r_{i k}^{j} v_{k}=0 \quad(1 \leq i \leq n, 1 \leq j \leq m)$.

$$
\sum_{j=1}^{k(\lambda)} u_{\lambda, j}-\sum_{j=1}^{l(\lambda)} \sum_{k=1}^{n} a_{\lambda, j, k}=0 \quad \text { for } \lambda \in \Lambda,
$$

where $a_{\lambda, j, k}=x_{k}\left(a_{\lambda, i}\right) \rho\left(a_{\lambda, 1} \cdots a_{\lambda, i-1}\right) v_{k}$, and

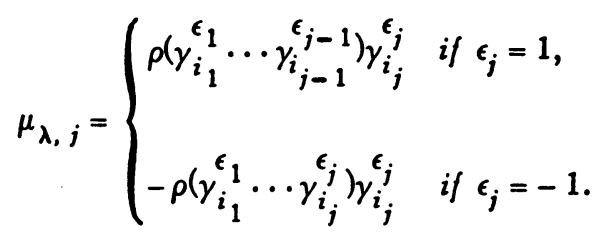

$\left(\right.$ Here $\left.\omega_{\lambda}=\gamma_{i_{1}}^{\epsilon_{1}} \cdots \gamma_{i_{k}(\lambda)}^{\epsilon_{k}(\lambda)}\right)$

(3.2) Now we prove the theorem of Nijenhuis and Richardson announced in [6].

Theorem 1 . Let $G$ be a compactly generated Lie group and $H$ any Lie group. Let $f_{0} \in \operatorname{Hom}(G, H)$. If $H^{1}\left(G, \mathcal{H}, \operatorname{Ad} \circ f_{0}\right)=0$, then $f_{0}$ is rigid.

Proof. We first identify the space $\operatorname{Hom}(G, H)$ with a closed subset of $\mathcal{H}^{n} \times H^{m}$ under the map $\epsilon$ in $\$ 2$, and let $f_{0}=\epsilon\left(f_{0}\right)=\left(Y_{1}^{0}, \ldots, Y_{n}^{0} ; b_{1}^{0}, \ldots, b_{m}^{0}\right)$. Next we identify the tangent linear space of $\mathcal{H}^{n} \times H^{m}$ at $f_{0}$ with that of $\mathcal{H}^{n} \times H^{m}$ at $(0, \ldots, 0,1, \ldots, 1)$ by the right translation.

On the other hand, we identify the tangent linear space of $\mathcal{H}^{n} \times H^{m}$ at $(0, \ldots, 0,1, \ldots, 1)$ with $\mathcal{H}^{n} \times \mathcal{H}^{m}$.

Let $\epsilon^{\prime}: Z^{1}\left(G, \mathcal{H}, \mathrm{Ad} \cdot f_{0}\right) \rightarrow \mathcal{H}^{n} \times \mathcal{H}^{m}$ be defined as in the beginning of this section with $V=\mathcal{H}$. Then a routine (but lengthy) computation shows that the tangent maps $d \Phi_{j}, d \psi_{i j}, d \Omega_{i j}$ and $d \chi_{\lambda}$ are given by the expressions on the 
left-hand sides of the equations (i), (ii), (iii) and (iv), respectively, of the lemma in (3.1). Hence from the lemma,

$$
\begin{aligned}
\epsilon^{\prime}\left(Z^{1}\left(G, \mathcal{H}, \text { Ad } \cdot f_{0}\right)\right)= & \left(\bigcap_{j} \operatorname{ker} d \Phi_{j}\right) \cap\left(\bigcap_{i, j} \operatorname{Ker} d \psi_{i j}\right) \\
& \cap\left(\bigcap_{i, j} \operatorname{ker} d \Omega_{i j}\right) \cap\left(\bigcap_{\lambda} \operatorname{Ker} d \chi_{\lambda}\right) .
\end{aligned}
$$

Now we define $\Lambda: H \rightarrow \mathcal{H}^{n} \times H^{m}$ by $\Lambda(b)=I_{b} \cdot f_{0} \in \operatorname{Hom}(G, H) \subset \mathcal{H}^{n} \times H^{m}$. Then it is easy to see that $\operatorname{Im}(d \Lambda)=\epsilon^{\prime}\left(B^{1}\left(G, \mathcal{H}, \mathrm{Ad} \cdot f_{0}\right)\right)$. Hence $H^{1}\left(G, \mathcal{H}, \operatorname{Ad} \cdot f_{0}\right)$ $=0$ implies that $\operatorname{Im}(d \Lambda)$ coincides with the intersection of the kernels of $d \Phi_{j}$, $d \psi_{i j}, d \Omega_{i j}$ and $d \chi_{\lambda}$. Hence by Lemma 1 of Weil [7], we can find a 1-neighborhood $U$ of $H$ so that $\Lambda(U)$ is open. Since $\Lambda(U) \subset \operatorname{Hom}(G, H)$, Int $(H) \cdot f_{0}$ is a neighborhood of $f_{0}$ in $\operatorname{Hom}(G, H)$.

To show that Int $(H) \cdot f_{0}$ is open, consider $f=I_{b} \cdot f_{0} \in \operatorname{Int}(H) \cdot f_{0}$. Then $H^{1}(G, \mathcal{H}, \mathrm{Ad} \cdot f) \cong H^{1}\left(G, \mathcal{H}\right.$, Ad $\left.\cdot f_{0}\right)=0$. Hence Int $(H) \cdot f$ is a neighborhood of $f$ and is contained in Int $(H) \cdot f_{0}$. Hence Int $(H) \cdot f_{0}$ is open, proving the rigidity of $f_{0}$.

4. Proof of the main result and application. In this section, we extend the main result of the preceding section to locally compact groups.

(4.1) We begin with the following special case:

Proposition. Let $G$ be a compact group and let $H$ be any Lie group. Then every $f_{0} \in \operatorname{Hom}(G, H)$ is rigid.

Proof. Let $K=\operatorname{Ker} f_{0}$. Then $G / K$ is a compact Lie group and $f_{0}$ induces $\hat{f}_{0} \in \operatorname{Hom}(G / K, H)$. Since $H$ is a Lie group, there exists an open 1-neighborhood $U$ of $H$ which contains no subgroups other than $\{1\}$.

Next we consider the basic neighborhood $W\left(K, U ; f_{0}\right)$. If $f \in W\left(K, U ; f_{0}\right)$, then $f(K) \subset U$, and hence $f(K)=\{1\}$ by the choice of $U$. Thus each $f \in W\left(K, U ; f_{0}\right)$ defines a unique $\hat{f} \in \operatorname{Hom}(G / K, H)$ so that $\hat{f} \cdot \pi=f$, where $\pi: G \rightarrow G / K$ is the canonical projection.

We now define $\sigma: W\left(K, U ; f_{0}\right) \rightarrow \operatorname{Hom}(G / K, H)$ by $\sigma(f)=\hat{f}$.

Clearly $\sigma$ is continuous and if $\pi^{*}=\operatorname{Hom}(\pi, 1): \operatorname{Hom}(G / K, H) \rightarrow \operatorname{Hom}(G, H)$ is the dual map induced by $\pi$, then $\sigma \cdot \pi^{*}=1$ on $\operatorname{Im} \sigma$ and $\pi^{*} \cdot \sigma=1$ on $W\left(K, U ; f_{0}\right)$. Hence $\pi^{*}: \operatorname{Im} \sigma \rightarrow W\left(K, U ; f_{0}\right)$ is a homeomorphism.

Since $G / K$ is a compact group, $H^{1}\left(G / K, \mathcal{H}, \mathrm{Ad} \cdot \sigma\left(f_{0}\right)\right)=(0)$. Hence, by the theorem in (3.2), $\sigma\left(f_{0}\right)=f_{0}$ is rigid. From this, it is immediate that $\pi^{*}\left(\right.$ Int $\left.(H), \sigma\left(f_{0}\right)\right)=\operatorname{Int}(H) \cdot f_{0}$ is a neighborhood of $f_{0}$, proving the rigidity of $f_{0}$.

Remark. The above proposition was proved in [3] by using an entirely different method. 
(4.2) The following lemma will be needed in (4.3).

Lemma. Let $M$ be a locally compact group and let $K$ be a closed subgroup of $M$ so that $M=M_{0} \cdot K$. If $K_{1}$ is an open subgroup of $K$, then $M_{0} \cdot K_{1}$ is open in $M$.

Proof. Choose a compact 1-neighborhood $U$ of $M$, and let $L$ be the subgroup of $M$ generated by $U$. As $L$ is open in $M, L J M_{0}$. Hence $L=M_{0}$. $(K \cap L)$ and $K \cap L$ is open in $K . M_{0} \cdot K_{1}$ would be open in $M$ if we can show that $M_{0} \cdot\left(K_{1} \cap L\right)$ is open in $M_{0} \cdot(K \cap L)=L$. Hence, replacing $K$ by $K \cap L$ if necessary, we may assume that $M$ (and hence $K$ ) is $\sigma$-compact.

Now choose a compact 1 -neighborhood $V$ in $K_{1}$. Since $K$ is $\sigma$-compact, we can write $K$ as $K=\bigcup_{i=1}^{\infty} V x_{i}$ with $x_{i} \in K$. Then $M=M_{0} \cdot K=\bigcup_{i=1}^{\infty} M_{0} \cdot V x_{i}$.

Since $M$ is locally compact, one of the $M_{0} V x_{i}$ has a nonvoid interior. Hence $M_{0} V$ has a nonvoid interior, which implies that $M_{0} K_{1}$ is open in $M_{\text {. }}$

(4.3) Now we are ready to prove the main theorem.

Theorem 2. Let $G$ be a compactly generated locally compact group and let $H$ be any Lie group. Let $f_{0} \in \operatorname{Hom}(G, H)$ with $H^{1}\left(G, \mathcal{H}, A d \cdot f_{0}\right)=0$. Then $f_{0}$ is rigid.

Proof. Let $K$ be the kernel of $f_{0^{\circ}}$. Then $G_{0} \cdot K$ is open in $G$. To see this, let $L$ be an open subgroup of $G$ containing $G_{0}$ with compact $L / G_{0}$. Then

$$
\left(G_{0} K\right) \cap L=G_{0} \cdot(K \cap L) .
$$

If $G_{0} \cdot(K \cap L)$ is open in $L, G_{0} K$ is open in $G$. Hence, replacing $G$ by $L$ if necessary, we may assume that $G / G_{0}$ is compact. For such a group, we can find a compact totally disconnected subgroup $C$ of $G$ such that $G=G_{0} \cdot C$. (See [3, Theorem 2.13, p. 42].) Let $\pi: G \rightarrow G / K$ be the natural projection. Then $\pi(G)=\pi\left(G_{0}\right) \pi(C)$. As $K$ is the kernel of $f_{0}, G / K$ is a Lie group. Hence $\pi(C)$ is finite and hence $\pi\left(G_{0}\right)$ is open in $\pi(G)=G / K$, proving that $G_{0} K / K$ in $G$ is open in $G / K$. Hence $G_{0} K$ is open.

Next we choose an open subgroup $K_{1}$ of $K$ containing $K_{0}$ so that $K_{1} / K_{0}$ is compact. Then applying the lemma to $M=G_{0} K$, we see that $G_{0} \cdot K_{1}$ is open in $M$ (and hence in $G$ ). Choose a compact subgroup $C_{1}$ of $K_{1}$ so that $K_{1}=$ $K_{0} \cdot C_{1}$. Then $G_{0} \cdot C_{1}\left(=G_{0} \cdot K_{1}\right)$ is open in $G$.

By a theorem of Iwasawa [2], $G_{0}$ contains the maximal compact connected normal subgroup $P$ so that $G_{0} / P$ is a Lie group. Then $G_{0} / K \cap P$ is also a Lie group. Since $K \cap P$ is a normal subgroup of $K$, the compact subgroup $C_{1}$ of $K$ as found above normalizes $K \cap P$.

Let $C=(K \cap P) \cdot C_{1}$. If $N$ is any closed normal subgroup of $G$ containing $C$, then $G / N$ is a Lie group. In fact, $G_{0} \cdot N$ is open in $G$ because $G_{0} \cdot N$ 
contains $G_{0} \cdot C_{1}$ which is open in $G$. Also $G_{0} / N \cap G_{0}$ is a Lie group because $N \cap G_{0}$ contains $K \cap P$. Thus it follows that $G / N$ is a Lie group.

Now let $i^{*}=\operatorname{Hom}(i, 1): \operatorname{Hom}(G, H) \rightarrow \operatorname{Hom}(C, H)$ be the dual map induced by the inclusion $i: C \rightarrow G$. Since $C$ is compact, $i^{*}\left(f_{0}\right)=f_{0} \mid C \in \operatorname{Hom}(C, H)$ is rigid by the proposition in (4.1). Hence Int $(H) \cdot i^{*}\left(f_{0}\right)$ is an open neighborhood of $i^{*}\left(f_{0}\right)$ and $W=\left(i^{*}\right)^{-1}\left(\operatorname{Int} H \cdot i^{*}\left(f_{0}\right)\right)$ is an open neighborhood of $f_{0}$ in Hom $(G, H)$. If $g \in W$, then there exists $b \in H$ so that $g(x)=h f_{0}(x) b^{-1}$ for all $x \in C$. From this, $\operatorname{Ker} G \cap C=\operatorname{Ker} f_{0} \cap C$. But $C \subseteq \operatorname{Ker} f_{0}=K$. Hence $C \subseteq \operatorname{Ker} g$ for all $g \in W$. Let $N$ be the intersection for all $\operatorname{Ker} g, g \in W$. Then $C \subseteq N$ and hence $G / N$ is a Lie group.

Let $\delta: G \rightarrow G / N$ be the natural projection and let $\delta^{*}=\operatorname{Hom}(\delta, 1)$ : $\operatorname{Hom}(G / N, H) \rightarrow \operatorname{Hom}(G, H)$ be the dual map of $\delta$. For each $g \in W$, there exists a unique $\hat{g} \in \operatorname{Hom}(G / N, H)$ so that $g=\hat{g} \cdot \delta^{*}(g)$. If we define $\sigma(g)=\hat{g}$, then $\sigma: W \rightarrow \operatorname{Hom}(G / N, H)$ is a continuous map and we have $\sigma \cdot \delta^{*}=1$ on $\operatorname{Im} \sigma, \delta^{*} \cdot \sigma$ $=1$ on $W$. Hence $\delta^{*}: \operatorname{Im} \sigma \rightarrow W$ is a homeomorphism. On the other hand, $H^{1}\left(G, \mathcal{H}\right.$, Ad $\left.\cdot f_{0}\right)=0$ implies that $H^{1}\left(G / N, \mathcal{H}\right.$, Ad $\left.\cdot \hat{f}_{0}\right)=0$.

Hence by the theorem in $\$ 3, f_{0}$ is rigid. That is, Int $(H) \cdot \hat{f}_{0}$ is an open neighborhood of $f_{0}$. It follows that $\delta^{*}\left(\operatorname{Int}(H) \cdot \hat{f}_{0} \cap \operatorname{Im} \sigma\right)$ is an open neighborhood of $\delta^{*}\left(\hat{f}_{0}\right)=f_{0}$ in $W$. From this, it is clear that Int $(H) \cdot f_{0}=\delta^{*}\left(\operatorname{Int}(H) \cdot f_{0}\right)$ is an open neighborhood of $f_{0}$ in $\operatorname{Hom}(G, H)$, proving that $f_{0}$ is rigid.

(4.4) Example. The theorem is false without the condition that $G$ is compactly generated. In fact, let $G$ be the quasi cyclic group $Z\left(p^{\infty}\right)$ with the discrete topology and let $H$ be the circle group. If $f_{0}$ is the zero map, then $H^{1}\left(G, \mathcal{H}, \operatorname{Ad} \cdot f_{0}\right)=\operatorname{Hom}(G, R)=0$. Hence $\operatorname{Hom}(G, H)$ would be a discrete group. However Hom $(G, H)=\hat{G}$, the character group of $G$, is well known to be the group of $p$-adic integers which is not discrete.

(4.5) Application. Using the main result, we characterize compactly generated groups which do not assume any nontrivial real characters.

Theorem. Let $G$ be a compactly generated locally compact group. Then the following are equivalent:

(1) There is no nontrivial continuous bomomorphism of $G$ into $R$, the addi. tive group of real numbers.

(2) The trivial homomorphism from $G$ into any Lie group $H$ is an isolated point in the space $\operatorname{Hom}(G, H)$.

Proof. (1) implies (2). (1) states that $\operatorname{Hom}(G, \Re)=0$. Let $H$ be any Lie group and let $f_{0}: G \rightarrow H$ be the trivial homomorphism. Then $\operatorname{Ad}_{H} \cdot f_{0}=0$. Hence $H^{1}\left(G, \mathcal{H}, \operatorname{Ad}_{H} \cdot f_{0}\right)=\operatorname{Hom}(G, \mathcal{H})=0$ because Hom $(G, \mathcal{R})=0$. Hence by the main result, $\left\{f_{0}\right\}$ is an open neighborhood of $f_{0}$, proving (2). 
(2) implies (1). First we note that $\operatorname{Hom}(G, \mathfrak{R}) \cong \operatorname{Hom}\left(G / G^{\prime}, \mathfrak{R}\right)$ where $G^{\prime}$ denotes the closure of the commutator subgroup of $G$. By (2), Hom $\left(G / G^{\prime}, R\right)$ is a discrete topological group.

On the other hand, $G / G^{\prime}$ is a compactly generated abelian group. Hence $G / G^{\prime}$ is isomorphic with the group of the form $R^{n} \times Z^{m} \times T$, where $T$ is the maximal compact subgroup. Hence $\operatorname{Hom}(G, R) \cong \operatorname{Hom}\left(G / G^{\prime}, \mathscr{R}\right) \cong \operatorname{Hom}\left(R^{n}, R\right)$ $\times \operatorname{Hom}\left(Z^{m}, \mathscr{R}\right)$. But Hom $\left(\Re^{n}, \mathscr{R}\right)$, and Hom $\left(Z^{m}, \mathscr{R}\right)$, are all connected. Since $\operatorname{Hom}(G, R)$ is discrete, it follows that $\operatorname{Hom}(G, \Re)=0$, proving $(1)$.

\section{BIBLIOGRAPHY}

1. C. Chevalley, Theory of Lie groups. Vol. I, Princeton Math. Series, vol. 8, Princeton Univ. Press, Princeton, N. J., 1946. MR 7, 412.

2. K. Iwasawa, On some types of topological groups, Ann. of Math. (2) 50 (1949), 507-558. MR 10, 679.

3. D. H. Lee, Supplements for the identity component in locally compact groups, Math. Z. 104 (1968), 28-49. MR $36 \# 6534$.

4. D. H. Lee and T. S. Wu, On conjugacy of homomorphisms of topological groups, Illinois J. Math. 13 (1969), 694-699. MR $40 \# 1525$.

5. G. D. Mostow, Cohomology of topological groups and solmanifolds, Ann. of Math. (2) 73 (1961), 20-48. MR 23 \# A2484.

6. A. Nijenhuis and R. Richardson, Jr., Deformations of homomorphisms of Lie groups and Lie algebras, Bull. Amer. Math. Soc. 73 (1967), 175-179. MR 34 \#4414.

7. A. Weil, Remarks on the cohomology of groups, Ann. of Math. (2) 80 (1964), 149157. MR 30 \#199.

DEPARTMENT OF MATHEMATICS, CASE WESTERN RESERVE UNIVERSITY, CLEVELAND, OHIO 44106 